\title{
Hypoglycaemia among Insulin-Treated Patients with Diabetes: Southeast Asia Cohort of IO HAT Study
}

Faruque Pathan, ${ }^{1}$ Su-Yen Goh, ${ }^{2}$ Achmad Rudijanto, ${ }^{3}$ Arvind Gadekar, ${ }^{4}$ Anand Jain, ${ }^{5}$ Nemencio Nicodemus Jr. ${ }^{6}$

\author{
${ }^{1}$ Department of Endocrinology, BIRDEM Hospital, Dhaka, Bangladesh \\ ${ }^{2}$ Singapore General Hospital, Singapore \\ ${ }^{3}$ Department of Internal Medicine, Faculty of Medicine, Brawijaya University, Malang, Indonesia \\ ${ }^{4}$ Novo Nordisk Pharma Operations (BAOS) Sdn. Bhd, Kuala Lumpur, Malaysia \\ ${ }^{5}$ Novo Nordisk Health Care AG, Zurich, Switzerland \\ ${ }^{6}$ College of Medicine, University of the Philippines Manila
}

\begin{abstract}
Objective: To provide real-world data on hypoglycaemia incidence in patients with type 1 (T1D) or type 2 diabetes (T2D) from the Southeast Asian cohort of the International Operations Hypoglycaemia Assessment Tool (IO HAT) study.

Methodology: IO HAT was a non-interventional, multicentre, 6-month retrospective and 4-week prospective study of hypoglycaemic events among insulin-treated adults with T1D or T2D, including four countries in Southeast Asia (Singapore, Philippines, Indonesia, and Bangladesh). Data were collected using a two-part self-assessment questionnaire (SAQ1 for retrospective and SAQ2 for prospective). The primary endpoint was the percentage of patients experiencing at least one hypoglycaemic event during the 4-week prospective observational period (ClinicalTrials.gov Identifier: NCT02306681).

Results: A total of 2594 patients completed SAQ1. Nearly all patients reported experiencing any hypoglycaemic event in the 4-week prospective period (T1D, 100\%; T2D, 97.3\%), with all patients reporting higher rates in the prospective versus retrospective period. Severe hypoglycaemia was also reported higher prospectively $(57.2 \%$ and $76.9 \%)$ than retrospectively (33.9\% and $12.2 \%$ ) in both T1D and T2D, respectively. Nocturnal hypoglycaemia was reported higher retrospectively than prospectively.
\end{abstract}

Conclusion: Incidence of any and severe hypoglycaemia in the Southeast Asian cohort of IO HAT was higher prospectively versus retrospectively, suggesting hypoglycaemia has previously been under-reported in this region.

Key words: hypoglycaemia, insulin, diabetes

\section{INTRODUCTION}

Patients with type 1 diabetes (T1D) and some with type 2 diabetes (T2D) rely on insulin therapy for survival and for glycaemic control. Prevalence of diabetes is escalating in every country, especially in developing countries, which has an impact on healthcare utilisation. ${ }^{1}$ Developing countries in Asia have the highest population of patients with diabetes in the world ${ }^{2-4}$ because of rapid urbanisation, unhealthy eating habits, sedentary lifestyles and increased life expectancy. ${ }^{5}$ A meta-analysis has shown a rapid increase in the prevalence of diabetes in Southeast Asia over the past 2 decades. 6 The overall prevalence of diabetes among adults in the region was $8.5 \%$ in 2015 , and this is estimated to rise to $10.7 \%$ by $2040 .{ }^{3}$ Furthermore, there is a high prevalence of impaired glucose tolerance (IGT) in Southeast Asia and the conversion rates from IGT to diabetes are faster in Southeast Asia than in other regions. ${ }^{1,7,8}$ Southeast Asian populations migrating from rural to urban areas that adopt unhealthy lifestyles are at a similar risk of developing diabetes as those who move from developing countries to more affluent countries. ${ }^{9}$ High consumption of carbohydrates (especially white rice) and inertia of physical activities in Asian populations, ${ }^{10}$ may lead to inefficient glycaemic control in those diagnosed with diabetes. An observational study that included patients from Indonesia, Thailand and Malaysia from the Southeast Asia region, found that $22 \%$ of patients with T1D and $36 \%$ of patients with T2D had never had glycated haemoglobin ( $\mathrm{HbA}_{1 \mathrm{c}}$ ) measurements. ${ }^{2}$ Despite the predicted increase in diabetes, a literature review, which was conducted with the primary purpose of determining the known incidence and impact of hypoglycaemia in Southeast Asia, highlighted that there are limited data regarding the incidence of hypoglycaemia in this region. ${ }^{11}$
ISSN 0857-1074 (Print) I ISSN 2308-118x (Online)

Printed in the Philippines

Copyright (C) 2018 by the JAFES

Received: September 13, 2017. Accepted: March 8, 2018

Published online first: April 4, 2018.

https://doi.org/10.15605/jafes.033.01.05
Corresponding Author: Prof. Md Faruque Pathan, MD

Head of the Department of Endocrinology

BIRDEM (Bangladesh Institute of Research \& Rehabilitation in Diabetes,

Endocrine and Metabolic Disorders) Hospital

122 Kazi Nazrul Islam Avenue, Shahbagh, Dhaka 1000, Bangladesh

Tel. No.: +880 2-8616641

Fax No.: $+880-2-9667812$

E-mail: pathan279@yahoo.com

ORCID iD: https://orcid.org/0000-0001-9163-2473 
Hypoglycaemia with insulin treatment is a major concern for both patients and healthcare providers and is a significant barrier to achieving good glycaemic control. ${ }^{12}$ Real-world data and literature on the incidence of hypoglycaemia in insulin-treated patients with diabetes are scarce. ${ }^{13}$ Results from the global Hypoglycaemia Assessment Tool (HAT) study from 24 countries worldwide showed that rates of hypoglycaemia among patients with insulin-treated T1D and T2D were higher than previously reported. ${ }^{14}$ The non-interventional International Operations HAT (IO HAT) study was designed to assess the incidence of hypoglycaemia in patients treated with insulin (premix, short-acting and long-acting) for T1D or T2D in Bangladesh, Colombia, Egypt, Indonesia, the Philippines, Singapore, South Africa, Turkey and United Arab Emirates. ${ }^{15}$

This publication presents the results from the Southeast Asian cohort of the IO HAT study (Bangladesh, Indonesia, Philippines and Singapore) and aims to determine, within this cohort, whether there are any differences in the incidence rates between retrospective and prospective reporting of hypoglycaemia.

\section{METHODOLOGY}

\section{Subjects}

Eligible patients (male or female patients 18 years or older with T1D or T2D treated with insulin for more than 12 months) were enrolled consecutively after attending a routine clinic appointment.

\section{Study design}

The methods for the IO HAT study have been previously published in detail, ${ }^{15}$ this sub-group analysis focuses on the Bangladesh, Indonesia, Philippines and Singapore cohorts from the IO HAT study. In summary, IO HAT was a non-interventional, multicentre, 6-month retrospective and 4-week prospective study of hypoglycaemic events in adult patients with T1D or T2D, treated with insulin for over 12 months (Figure 1). Retrospective cross-sectional and prospective observational evaluations took place using a two-part self-assessment questionnaire (SAQ1 and SAQ2) and the patient diary (provided at baseline and used to aid prospective reporting). There were no interventions or specific treatments recommended or provided to patients as a result of participating in this study. The trial protocol, any amendments, informed consent form and any other written information or documents, as required, were submitted to local institutional review board/ethics committees. The trial was performed in accordance with the Declaration of Helsinki and International Conference on Harmonisation of Technical Requirements for Registration of Pharmaceuticals for Human Use Good Clinical Practice guidelines.

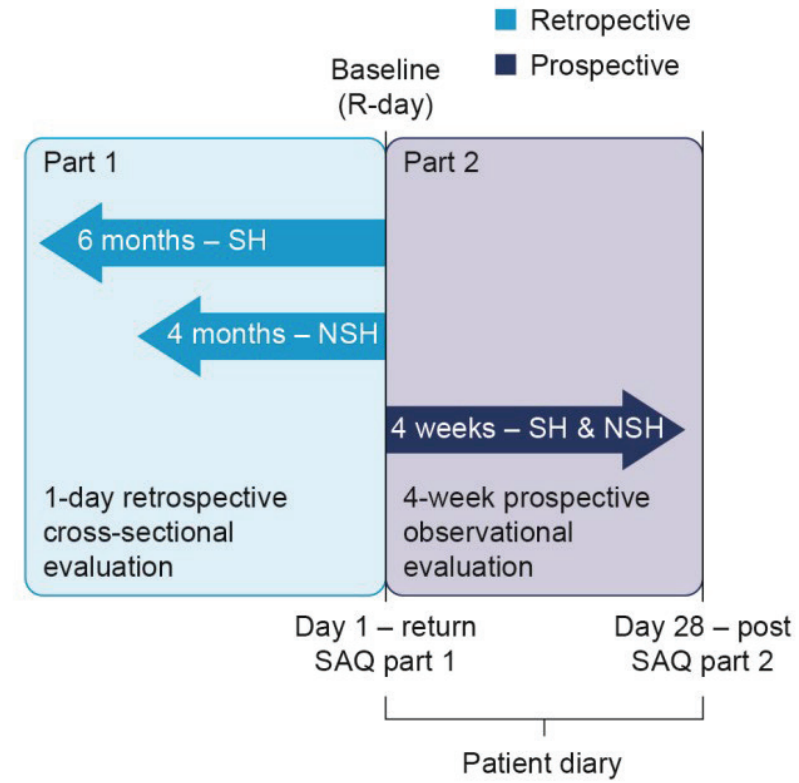

$\mathrm{NSH}$, non-severe hypoglycaemia; SAQ, self-assessment questionnaire; $\mathrm{SH}$, severe hypoglycaemia; T1D, type 1 diabetes; T2D, type 2 diabetes

\section{Figure 1. Study design. ${ }^{15}$}

Categories of hypoglycaemia recorded in the questionnaire and patient diary included severe hypoglycaemia (defined, based on the American Diabetes Association [ADA] definition from 2005, as any hypoglycaemic event requiring assistance of another person to administer carbohydrate, glucagon or other resuscitative actions ${ }^{16}$ ) any hypoglycaemia (the sum of non-severe hypoglycaemia [any event managed by the patient alone] and severe hypoglycaemia) and nocturnal hypoglycaemia (any event occurring between midnight and 06:00 am); the reported hypoglycaemia events did not need to be accompanied by a confirmatory blood glucose measurement. Patient knowledge of hypoglycaemia was evaluated by assessing whether their definition was consistent with the ADA definition of hypoglycaemia ${ }^{16}$ and if they knew what hypoglycaemia was before being provided with the informed consent form. Hypoglycaemia unawareness was evaluated using a previously validated question, ${ }^{17}$ 'Do you have symptoms when you have a low sugar level?', where the responses 'always,' 'usually,' 'occasionally' and 'never' represent increasing degrees of hypoglycaemia unawareness.

\section{Endpoints}

The primary objective for the IO HAT study was to assess the percentage of patients experiencing at least one hypoglycaemic event during the 4-week prospective observational period.

Key secondary objectives included: incidence of various types of hypoglycaemic episodes; the difference in the reported incidence of severe, non-severe, nocturnal and any hypoglycaemic episodes in the retrospective and 


\begin{tabular}{|c|c|c|}
\hline Characteristic & T1D (N=154) & T2D $(\mathrm{N}=2,440)$ \\
\hline Age (years) & $33.2(12.5)$ & $57.2(11.2)$ \\
\hline Median & 30.0 & 58.0 \\
\hline Upper quartile, Lower quartile & $40.0,24.0$ & $65.0,50.0$ \\
\hline Male/female (\%) & $44.8 / 55.2$ & $42.7 / 56.7$ \\
\hline \multicolumn{3}{|l|}{ Country, $\mathrm{n}$} \\
\hline Bangladesh & 25 & 1154 \\
\hline Philippines & 62 & 609 \\
\hline Indonesia & 17 & 357 \\
\hline Singapore & 50 & 320 \\
\hline Duration of diabetes (years) & $13.6(9.9)$ & $13.2(8.1)$ \\
\hline Median & 12.0 & 12.0 \\
\hline Upper quartile, lower quartile & $18.0,6.0$ & $18.0,7.0$ \\
\hline Duration of insulin use (years) & $12.5(9.7)$ & $5.5(4.9)$ \\
\hline Median & 10.0 & 4.0 \\
\hline Upper quartile, lower quartile & $16.0,5.0$ & $7.0,2.0$ \\
\hline BMI $\left(\mathrm{kg} / \mathrm{m}^{2}\right)$ & $22.4(3.7)$ & $26.4(4.8)$ \\
\hline Median & 22.0 & 25.7 \\
\hline Upper quartile, lower quartile & $24.9,19.7$ & $28.7,23.5$ \\
\hline $\mathrm{HbA}_{1 \mathrm{c}}(\%)$ & $8.4(1.8)$ & $8.7(1.9)$ \\
\hline FBG (mmol/l) & $8.8(4.7)$ & $8.6(3.5)$ \\
\hline PPG (mmol/l) & $10.2(4.5)$ & $11.4(4.0)$ \\
\hline Weight (kg) & $57.9(11.9)$ & $67.3(13.1)$ \\
\hline Median & 56.5 & 66.0 \\
\hline Upper quartile, lower quartile & $65.0,49.0$ & $74.0,59.0$ \\
\hline Height $(\mathrm{cm})$ & $160.6(9.9)$ & $159.7(8.6)$ \\
\hline Median & 159.5 & 160.0 \\
\hline Upper quartile, lower quartile & $168.0,153.0$ & $165.0,153.0$ \\
\hline \multicolumn{3}{|l|}{ Previous medical illnesses ( $\%$ patients) } \\
\hline Neuropathy & 21.4 & 42.2 \\
\hline Retinopathy & 27.9 & 38.2 \\
\hline Nephropathy & 8.4 & 21.7 \\
\hline Peripheral vascular disease & 14.3 & 19.1 \\
\hline Angina & 4.5 & 12.6 \\
\hline Myocardial infarction & 0.6 & 9.8 \\
\hline None & 55.8 & 29.6 \\
\hline \multicolumn{3}{|l|}{ Method of diabetes treatment [n(\%)] } \\
\hline Short-acting insulin & $6(3.9)$ & $148(6.1)$ \\
\hline Long-acting insulin & $8(5.2)$ & $467(19.1)$ \\
\hline Pre-mix insulin & $50(32.5)$ & $1069(43.8)$ \\
\hline Both short and long acting insulin & $87(56.5)$ & $647(26.5)$ \\
\hline Both short acting and pre-mix insulin & $2(1.3)$ & $70(2.9)$ \\
\hline Both long acting and pre-mix insulin & $0(0.0)$ & $32(1.3)$ \\
\hline Short and long acting and pre-mix insulin & $1(0.6)$ & $1(0.0)$ \\
\hline Missing & $0(0.0)$ & $6(0.2)$ \\
\hline
\end{tabular}

prospective periods; the relationship between demography, duration of diabetes, duration of insulin therapy and incidence of hypoglycaemic episode; use of healthcare resources; and patient behaviours in response to hypoglycaemia.

\section{Statistical analyses}

Statistical tests were two-sided and exploratory, with the criterion for statistical significance set at $p<0.05$. There was no adjustment for multiple comparisons, but $p$-values were interpreted conservatively, i.e. $p$-values from 0.01 to 0.05 were taken to indicate modest evidence of a difference and $p$-values of less than 0.01 were taken to indicate moderate evidence of a difference.

For the primary endpoint, the percentage of patients who experienced at least one hypoglycaemic episode during the 4-week prospective observational period among T1D or T2D was calculated together with the confidence interval for this percentage. For secondary endpoints, the incidence of various types of hypoglycaemia was calculated as the number of events per patient year.
Sample size was determined assuming a worst case scenario proportion of patients $(=50 \%)$ reporting at least one hypoglycaemic episode during the 4-week prospective observation period, and that the range of the $95 \%$ confidence interval was less than 3 percentage points for the total cohort $(\mathrm{N}=6000)$. The sample size was calculated using 50\%, which represents the largest degree of variance.

\section{RESULTS}

\section{Patient characteristics}

Patient characteristics are described in Table 1. Of the 7289 patients enrolled in the IO HAT study, 2594 (T1D, $\mathrm{n}=154$; T2D, $\mathrm{n}=2440$ ) were from Southeast Asia (Bangladesh [ $\mathrm{n}=1179]$, Indonesia [ $\mathrm{n}=374]$, the Philippines $[\mathrm{n}=671]$ and Singapore [n=370]; Table 1). Patients included in the full analysis set completed SAQ1 (2594 [T1D, n=154; T2D, n=2440]). Of these, 2483 completed SAQ2 and were included in the completer analysis set (T1D, n=138; T2D, n=2345). 
Patients with T2D were older than those with T1D, with a mean age of 57.2 years compared with 33.2 years. The average duration of diabetes was similar in both patients with T1D (13.6 years) and T2D (13.2 years). On average, patients with T1D had a longer average duration of insulin use (12.5 compared with 5.5 years for patients with T2D) and lower average $\mathrm{HbA}_{1 \mathrm{c}}(8.4 \%[ \pm 1.8])$ than those with T2D $(8.7 \%$ [ \pm 1.9$])$. Most patients (87 patients [56.5\%]) with T1D were on mixed insulin regimens (both short- and long-acting insulin), followed by pre-mix (50 patients [32.5\%]), long-acting insulin (eight patients [5.2\%]) and short-acting (six patients [3.9\%]). Only two patients (1.3\%) were on a mixed regimen of short-acting and pre-mix insulin and one patient $(0.6 \%)$ was on a mixed regimen of short- and long-acting and pre-mix insulins. Most patients (1069 patients [43.8\%]) with T2D used pre-mix insulins, followed by $647(26.5 \%)$ patients on a mixed regimen of short- and long-acting insulin and 467 patients [19.1\%] on long-acting insulin.

\section{Frequency of hypoglycaemia}

\section{Any hypoglycaemia}

Most patients (T1D, 100\%; T2D, 97.3\%) reported experiencing an hypoglycaemic event during the 4-week prospective period (Figure 2). Lower rates of any hypoglycaemia were reported retrospectively compared with prospectively in patients with T1D (rate ratio [RR] $1.68, p<0.001$ ) or T2D (RR 1.85, $p<0.001$; Figure 2). For any hypoglycaemia, the rates per-patient year (PPY) during the retrospective period for T1D and T2D were $33.9(95 \%$ CI: 30.68, 37.44) and 12.2 (95\% CI: 11.69, 12.70), respectively. In the prospective period, rates for T1D and T2D were higher (57.1 [95\% CI: 52.63, 61.83] PPY and 22.6 [95\% CI: 21.94, 23.35] PPY, respectively) than in the retrospective period.

\section{Nocturnal hypoglycaemia}

During the 4 -week prospective period, $34.8 \%$ of patients with T1D and $11.7 \%$ of patients with T2D reported nocturnal hypoglycaemia. Higher rates of nocturnal hypoglycaemia were reported in the 6-month retrospective period than in the prospective period in both patients with T1D (RR 0.87, $p=0.459$ ) and T2D (RR 0.71, $p=0.003$; Figure 2). In patients with $\mathrm{T} 1 \mathrm{D}$ and $\mathrm{T} 2 \mathrm{D}$, rates of nocturnal hypoglycaemia in the retrospective period were 12.5 (95\% CI: 10.55, 14.75) PPY and 3.9 (95\% CI: 3.62, 4.19) PPY, respectively. Prospectively, lower rates of nocturnal hypoglycaemia were reported for both patients with T1D (11.2 [95\% CI: 9.23, 13.36] PPY) and T2D (2.9 [95\% CI: 2.61, 3.12] PPY).

\section{Severe hypoglycaemia}

During the 4 -week prospective period, $57.2 \%$ of patients with T1D and $76.9 \%$ of patients with T2D reported severe hypoglycaemia. Lower rates of severe hypoglycaemia were reported retrospectively compared with prospectively in patients with T1D (RR 1.67, $p=0.033$ ) or T2D (RR 4.11, $p<0.001$; Figure 2). In patients with T1D, rates a)

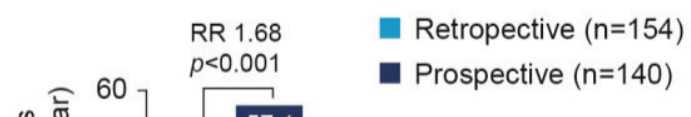

Patients with 64.1100 .0 hypoglycaemia (\%) Any

b)

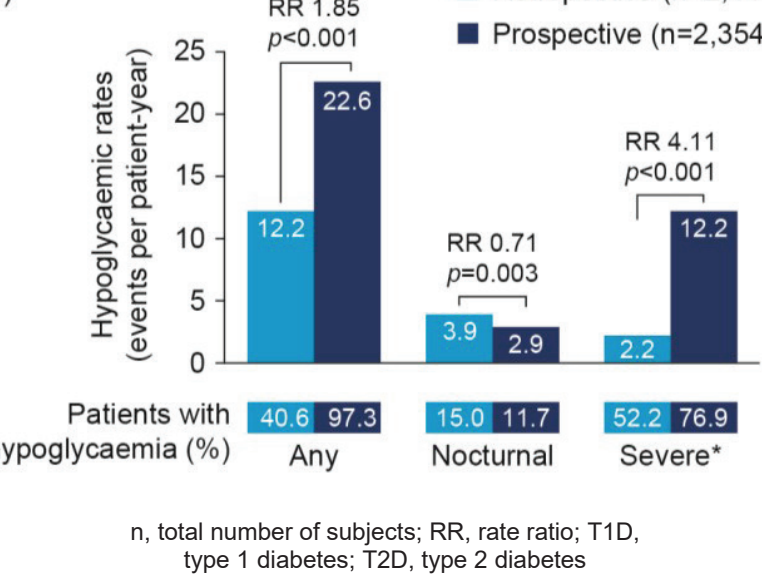

Figure 2. Retrospective and prospective hypoglycaemia rates in T1D (a) and T2D (b) 'Any' and 'Nocturnal' based on 4-week period for both retrospective and prospective analyses. *Retrospective data based on 6-month period and prospective data based on 4-week period.

of severe hypoglycaemia in the retrospective period were 3.8 [95\% CI: 3.39, 4.32] PPY and in T2D, 2.2 [95\% CI: 2.08, 2.25] PPY. Hypoglycaemia rates were higher in the prospective period for both patients with T1D (12.7 [95\% CI: 10.61, 15.00] PPY) and T2D (12.2 [95\% CI: 11.68, 12.72] PPY).

\section{Impact of hypoglycaemia on the medical system}

In the retrospective period 6 months before baseline, 7.1 and $7.3 \%$ of patients with T1D and T2D, respectively, had a hypoglycaemic event that led to hospital admission. During this time, $9.2 \%$ of patients with T1D attended additional clinical appointments as a result of a hypoglycaemic event and $7.8 \%$ made additional telephone contacts with a healthcare provider. In T2D, 6.8\% of patients who experienced a hypoglycaemic event made additional telephone calls and 6.2\% attended additional clinical appointments. In the 4-week prospective period, the most common effect on the medical system was making additional telephone contacts in both patients with T1D (4.3\%) and T2D (5.7\%), followed by attending additional clinical appointments $(2.9 \%$ for patients with T1D and $1.7 \%$ for T2D) and hospital admission $(0.7 \%$ for patients with T1D and 1.2\% for T2D). 


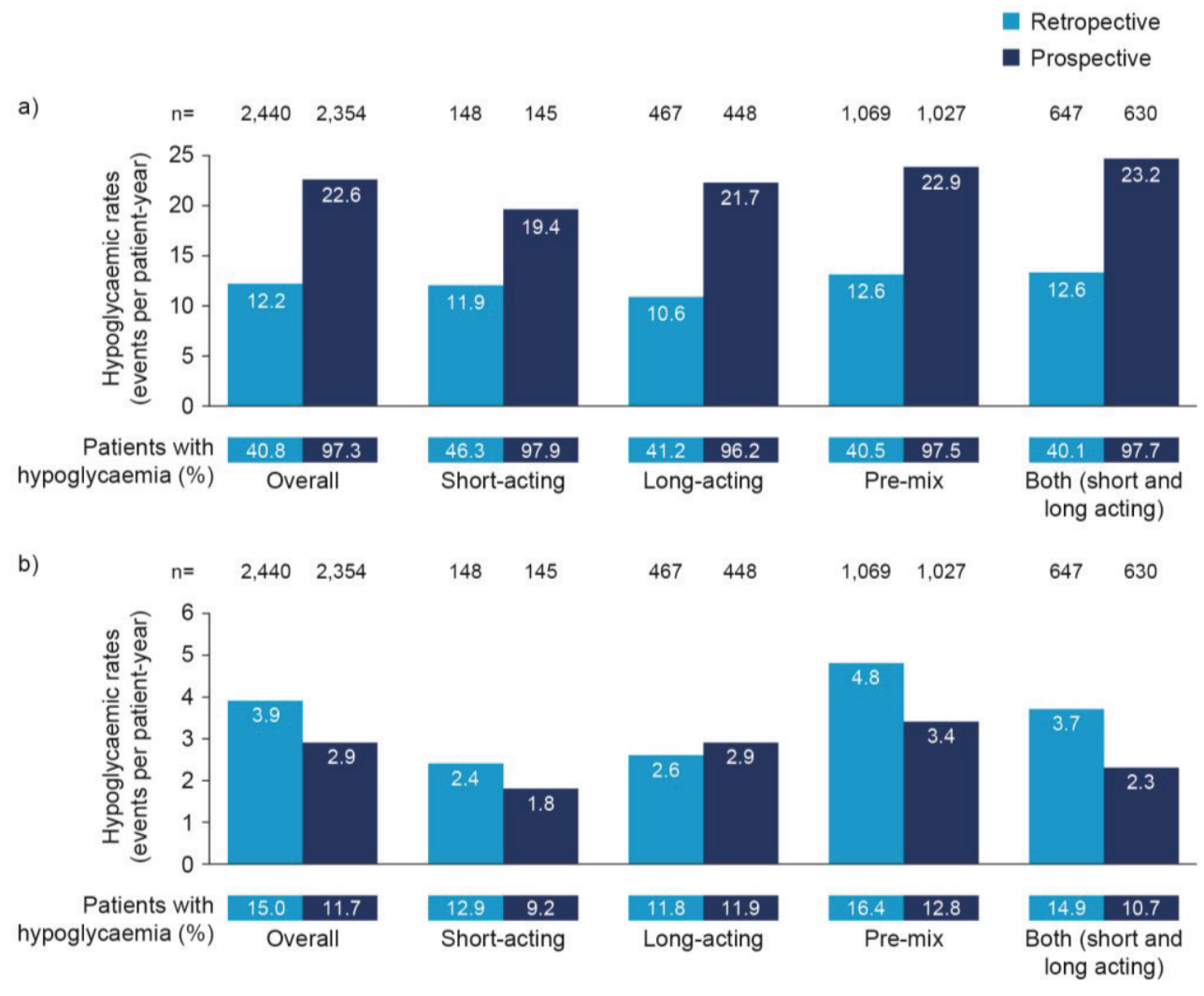

$\%$, percentage; $n$, total number of subjects; T2D, type 2 diabetes

Figure 3. Estimated rate of any (a) and nocturnal (b) hypoglycaemic events by insulin regimen in T2D.

\section{Rates of hypoglycaemia by insulin regimen}

Lower rates of any hypoglycaemia were reported retrospectively than prospectively with all insulin regimens in both patients with T1D and T2D (Figure 3a). In patients with T1D, lower rates of nocturnal hypoglycaemia were reported retrospectively than prospectively among those treated with short-acting (4.4 compared with 10.4 PPY, respectively) and long-acting (3.3 compared with 19.6 PPY, respectively) insulin regimens. Patients on pre-mix or mixed (both short- and long-acting) insulin reported higher rates of nocturnal hypoglycaemia retrospectively than prospectively (pre-mix: 15.7 compared with 13.3 PPY, respectively; mixed: 12.3 compared with 9.5 PPY, respectively). In patients with T2D, nocturnal hypoglycaemia was reported at a higher rate retrospectively than prospectively in patients treated with all insulin regimens except long-acting insulin (Figure $3 b$ ). Lower rates of severe hypoglycaemia were reported retrospectively than prospectively with all insulin regimens in both patients with T1D and T2D, with lower rates of hypoglycaemia in patients on long-acting insulin regimens.

In patients with $\mathrm{T1D}$, patients on short-acting insulin had the lowest rates of any hypoglycaemia in the prospective period (33.9 PPY). The highest rates of hypoglycaemia were reported by patients on a mixed insulin regimen (both short- and long-acting insulin; 61.3 PPY). In patients with T2D, rates of any and nocturnal hypoglycaemia (Figure 3) were also lowest with short-acting insulin regimens during the prospective period (19.4 and 1.8 PPY, respectively; Figure 3). The highest rates of any hypoglycaemia reported prospectively were in those on a mixed insulin regimen (short- and long-acting insulin; 23.2 PPY), followed by those on a pre-mix insulin regimen (22.9 PPY).

\section{Rates of hypoglycaemia by duration of diabetes and insulin therapy}

In the retrospective period, patients with T1D who had diabetes for between 12 and 17 years had the highest rates of any hypoglycaemia (40.5 PPY). In patients with T2D rates of hypoglycaemia increased with increasing duration of diabetes. Rates of nocturnal hypoglycaemia generally increased with diabetes duration for both patients with T1D and T2D, with peaks in those who had diabetes for between 12 and 17 years for T1D and between 7 and 11 years for T2D. Rates of severe hypoglycaemia generally increased with duration of diabetes for both patients with T1D and T2D. In the prospective period, for patients with T1D or T2D, rates of any, nocturnal and severe hypoglycaemia generally increased with increasing duration of diabetes.

Patients with T1D who had received insulin therapy for between 5 and 9 years reported the highest rates of any hypoglycaemia in the retrospective period. Patients who had received insulin therapy for over 10 years had the highest rates of nocturnal and severe hypoglycaemia. In 


\begin{tabular}{|c|c|c|c|c|}
\hline Perspective & \multicolumn{2}{|l|}{ T1D (N=154) } & \multicolumn{2}{|l|}{ T2D $(\mathrm{N}=2,440)$} \\
\hline Knew what hypoglycaemia was before reading definition provided [n/ $\mathrm{N}$ total (\%)] & \multicolumn{2}{|l|}{$148 / 154(96.1)$} & \multicolumn{2}{|l|}{$2046 / 2390(85.6)$} \\
\hline Defined hypoglycaemia on basis of [n/N total $(\%)]$ & \multirow{2}{*}{\multicolumn{2}{|c|}{$89 / 154(57.8)$}} & & \\
\hline Symptoms only & & & \multicolumn{2}{|l|}{$1144 / 2440(46.9)$} \\
\hline Blood glucose measurements only & \multicolumn{2}{|l|}{$6 / 154(3.9)$} & \multicolumn{2}{|l|}{$134 / 2440(5.5)$} \\
\hline Either & \multicolumn{2}{|l|}{$15 / 154(9.7)$} & \multicolumn{2}{|l|}{$227 / 2440(9.3)$} \\
\hline Both & \multicolumn{2}{|l|}{$36 / 154(23.4)$} & \multicolumn{2}{|l|}{$500 / 2440(20.5)$} \\
\hline \multicolumn{5}{|l|}{ Hypoglycaemia awareness (\%) } \\
\hline Normal & \multicolumn{2}{|l|}{48.7} & \multicolumn{2}{|l|}{39.6} \\
\hline Impaired & \multicolumn{2}{|l|}{46.8} & \multicolumn{2}{|l|}{44.1} \\
\hline Severely impaired & \multicolumn{2}{|l|}{0.6} & \multicolumn{2}{|l|}{10.9} \\
\hline \multicolumn{5}{|l|}{ Fear of hypoglycaemia (\%) } \\
\hline $0=$ no fear & \multicolumn{2}{|l|}{14.3} & \multicolumn{2}{|l|}{18.6} \\
\hline 1 & \multicolumn{2}{|l|}{3.2} & \multicolumn{2}{|l|}{7.3} \\
\hline 2 & \multicolumn{2}{|l|}{5.2} & \multicolumn{2}{|l|}{9.6} \\
\hline 3 & \multicolumn{2}{|l|}{7.8} & \multicolumn{2}{|l|}{10.6} \\
\hline 4 & \multicolumn{2}{|l|}{9.1} & \multicolumn{2}{|l|}{9.3} \\
\hline 5 & \multicolumn{2}{|l|}{19.5} & \multicolumn{2}{|l|}{16.1} \\
\hline 6 & \multicolumn{2}{|l|}{5.8} & 7.7 & \\
\hline 7 & 11.7 & & 6.4 & \\
\hline 8 & 11.0 & & 5.9 & \\
\hline 9 & 3.2 & & 2.8 & \\
\hline $10=$ absolutely terrified & 9.1 & & 5.7 & \\
\hline Impact of hypoglycaemic events on the medical system & Retrospective & Prospective & Retrospective & Prospective \\
\hline $\begin{array}{l}\text { Number of hospital admission days as a result of hypoglycaemia } \\
\text { [n, mean (SD)] }\end{array}$ & $140,0.1(0.5)$ & $138,0.0(0.1)$ & $1947,0.4(2.3)$ & $2273,0.0(0.5)$ \\
\hline Number of additional clinic appointments attended [n, mean (SD)] & $13,1.8(1.3)$ & $4,1.3(0.5)$ & $120,1.7(1.2)$ & $35,1.3(0.6)$ \\
\hline Number of additional telephone contacts made [n, mean (SD)] & $9,1.8(1.6)$ & $6,2.0(1.6)$ & $123,2.3(2.1)$ & $123,1.6(1.4)$ \\
\hline
\end{tabular}

the prospective period, patients with T1D who had received insulin therapy for over 10 years reported the highest rates of any, nocturnal and severe hypoglycaemia. Patients with T2D who had received insulin therapy for over 10 years had the highest rates of any, nocturnal and severe hypoglycaemia in the retrospective and prospective periods (compared with those who had received insulin therapy for 1 to $<3,3$ to $<5$ and 5 to $<10$ years).

\section{Rates of hypoglycaemia by frequency of blood glucose monitoring}

In both patients with T1D and T2D, rates of any hypoglycaemia increased with each quartile, and were highest in the upper quartile of frequency of blood glucose monitoring in both the retrospective and prospective periods. In patients with T2D, the same trend was seen for nocturnal and severe hypoglycaemia rates.

\section{Hypoglycaemic events recorded in the patient diary}

Of those who completed the patient diary (154 in T1D, 2440 in T2D) 36 patients (25.9\%) with T1D and 158 (7.0\%) patients with T2D had a confirmed hypoglycaemic event recorded in their diary.

\section{Impact of hypoglycaemia on work/school}

During the retrospective period, the impact of hypoglycaemia was higher on patients with T1D than T2D. In patients with $\mathrm{T1D}$, arriving late to work/studies was the most common impact of hypoglycaemia on work/school, retrospectively reported by $22.1 \%$ of patients, followed by taking leave (17.3\%) and leaving early (13.5\%). In patients with T2D, leaving early from work was reported by $10.4 \%$ of patients, followed by taking leave $(10.1 \%)$ and arriving late to work (7.6\%). Of those patients who held a driver's licence (T1D, n=50; T2D, n=580), six (12.0\%) with T1D and $25(4.3 \%)$ with T2D experienced a hypoglycaemic event whilst driving in the past 6 months.

In the 4-week prospective period, arriving late to work/studies was the most common impact of hypoglycaemia on work/school, reported by $10.0 \%$ of patients with T1D, followed by taking leave $(4.4 \%)$ and leaving early from work $(2.2 \%)$. In patients with T2D, taking leave was reported by $3.2 \%$ of patients, with the next most common impacts being leaving early $(2.8 \%)$ and arriving late $(2.1 \%)$.

\section{Patient perspectives}

Patient perspectives on hypoglycaemia are described in Table 2. In T1D and T2D, 96.1\% and $85.6 \%$ of patients knew what hypoglycaemia was before being provided with the definition in SAQ1. The most common method that patients used to define hypoglycaemia was based on symptoms alone $(57.8 \%$ in T1D and $46.9 \%$ in T2D). More patients with T1D had normal hypoglycaemia awareness $(48.7 \%)$ than those with T2D (39.6\%). Patients with T2D had more hypoglycaemia unawareness than those with T1D (55\% of patients with T2D had impaired or severely impaired awareness compared with $47.4 \%$ for T1D). When answering the question 'How often do you have symptoms when you have a low blood sugar measurement' $44.1 \%$ of patients with T2D and $46.8 \%$ in T1D, answered 'occasionally,' indicating impaired awareness and 10.9\% answered 'never' indicating severely impaired awareness $(\mathrm{T} 1 \mathrm{D}=0.6 \%)$. Of those patients with impaired awareness, $46.9 \%$ with T1D and $45.4 \%$ with T2D experienced severe hypoglycaemia in the 6-months before baseline. 
Patients reported a moderate fear of hypoglycaemia, with mean scores out of ten of five for T1D and four for T2D (where one indicated not afraid at all and ten, absolutely terrified). Retrospectively, the most common patient actions resulting from a hypoglycaemic event in T1D and T2D were requiring medical assistance $(53.9 \%$ and $39.9 \%$, respectively), consulting their doctor/nurse $(51.3 \%$ and $38.8 \%$ respectively) and increasing calorie intake $(40.9 \%$ and $27.3 \%$ respectively). In the 4 -week prospective period, increasing calorie intake, requiring medical assistance and consulting their doctor or nurse were the most common actions taken by patients with T1D and T2D.

\section{Associations between hypoglycaemia and continuous or predictor variables}

No association was identified between $\mathrm{HbA}_{1 c}$ level and any, nocturnal or severe hypoglycaemia.

\section{DISCUSSION}

The IO HAT study was a multicentre, international, 6month retrospective and 4-week prospective study with a two-part SAQ that investigated the prevalence of hypoglycaemia in insulin-treated adults with T1D or T2D.

Results from the Southeast Asia cohort of the IO HAT study show that the proportion of patients who experienced at least one hypoglycaemic event was higher in the prospective period than the retrospective period for both T1D and T2D. Patients with T1D reported higher incidence rates than those with T2D in both periods for any, nocturnal and severe hypoglycaemia. Higher rates of nocturnal hypoglycaemia were reported in the retrospective period than in the prospective period, for both patients with T1D and T2D.

The higher rates of any and severe hypoglycaemia reported during the prospective period were also seen in the overall IO HAT study. ${ }^{15}$ Despite a high proportion of patients who knew what hypoglycaemia was before learning the definition during the study (T1D 96.1\% and T2D 85.6\%), the most common way patients defined hypoglycaemia was based on symptoms only and nearly half of patients had impaired hypoglycaemia awareness. One review identified a high proportion of diabetes unawareness in Indonesia and suggested that patients in Southeast Asia may be poorly educated about hypoglycaemia and its management. ${ }^{11}$ The reduced level of hypoglycaemia and diabetes awareness in this region may have contributed to the lower reporting of hypoglycaemic events by patients in the retrospective period of this study. However, the study demonstrated that when patients are properly educated about hypoglycaemia and its manifestations, patients are able to recognise the signs of hypoglycaemia themselves.

This cohort had a small number of patients with T1D $(n=154) . T 1 D$ is rare in Asian populations and data collection is not as systematic as in Western countries. ${ }^{1}$
Most Southeast Asian countries do not have established registries for patients with $\mathrm{T} 1 \mathrm{D}$, which may contribute to the lack of data on T1D and hypoglycaemia in this region. ${ }^{1}$ The little information available does however suggest that incidence of T1D is rising in Asia. ${ }^{1}$

Patient responses to hypoglycaemia increase healthcare utilisation in Southeast Asia. This may be due to seizures induced by hypoglycaemia, which can eventually lead to coma and death. ${ }^{18}$ Hypoglycaemia may also result in patients missing doses and reactively adjusting their medication plan. ${ }^{19}$ In the present study, most patients who experienced hypoglycaemia made contact with their doctor or nurse and required a form of medical assistance in T1D and T2D, both retrospectively and prospectively.

A study in Bangladesh found that patients with diabetes had twice the number of inpatient treatment days than those without diabetes. They also had 1.3-fold more outpatient visits and 9.7-fold more medications than those without diabetes, and the cost of their healthcare was 6.1fold higher. ${ }^{20}$ There is a lack of structured care management and integrated healthcare policies in the Southeast Asia region, ${ }^{1,9}$ differences in diabetes healthcare available in rural, compared with urban areas, must be taken into consideration.

Some patients increased their calorie intake to overcome the effects of hypoglycaemia in both the retrospective and prospective periods (retrospectively: T1D, 40.9\% and T2D, $27.3 \%$; prospectively: $\mathrm{T} 1 \mathrm{D}, 36.2 \%$ and $\mathrm{T} 2 \mathrm{D}, 16.8 \%$ ), this is in line with results from the global HAT study. ${ }^{21}$ If patients manage hypoglycaemia themselves by increasing calorie intake, their $\mathrm{HbA}_{1 c}$ during clinic visits may be elevated and may result in their healthcare practitioner (HCP) increasing or adding more antidiabetes agents to their treatment. More frequent monitoring and clinic visits with a duration of self-monitoring might be advised to try and achieve better glucose control. Continuous glucose monitoring has been shown to be cost-effective in patients with T1D with intensive insulin therapy ${ }^{22}$ and in patients with T2D on oral antidiabetic drugs some of the increased costs associated with self-monitoring blood glucose are offset by reductions in diabetes complication costs..$^{23,24}$

Pre-mix insulin is the most common method of insulin treatment in Southeast Asia and this is reflected in the results from this cohort with $32.5 \%$ of patients with T1D and $43.8 \%$ of patients with $\mathrm{T} 2 \mathrm{D}$ on this insulin regimen at baseline. In the retrospective period, patients on pre-mix insulin reported the highest rates of any, nocturnal and severe hypoglycaemia in T1D and for any and nocturnal hypoglycaemia in T2D. This is in contrast to the overall IO HAT study, as in patients with T1D, the highest rates of any hypoglycaemia retrospectively and prospectively were reported with short-acting insulin. ${ }^{15}$ In patients with $\mathrm{T} 2 \mathrm{D}$, highest rates of any hypoglycaemia were reported with a mixed insulin regimen (both short- and long-acting 
insulin) retrospectively, whereas short-acting, premix and mixed regimens (both short- and long-acting insulins) had the highest rates reported prospectively. ${ }^{15}$ The high hypoglycaemia rates identified with pre-mix insulin in Southeast Asia are similar to previous data. ${ }^{11}$ Traditionally, the Asian diet is high in carbohydrates, and so pre-mix insulins are a suitable choice as they target post-prandial hyperglycaemia. ${ }^{25}$ However, newer insulin analogues can provide more stable glycaemic control and effective reduction of post-prandial hyperglycaemia.

Hypoglycaemia is known to have an impact on patients' productivity. Productivity of patients in this region is clearly affected with $17.3 \%$ of patients with T1D and $10.1 \%$ of patients with $\mathrm{T} 2 \mathrm{D}$ in this study arriving late to work/studies. In Singapore, prevalence of T2D in adults is projected to rise from $7.3 \%$ in 1990 to $15 \%$ in $2050 . .^{5}$ These data suggest that this rise will have an effect on the workforce - there were 97,600 patients working and living with T2D in Singapore in 1990, which is expected to increase to 321,600 by $2050 .^{5}$ Singapore's combined Chinese, Indian and Malaysian population is representative of Asia as a whole, which makes it a useful test for healthcare research. ${ }^{5}$ The trends observed in this study could be used to predict the future impact of hypoglycaemia on the whole of Asia. If the pattern predicted is observed over time, this may have a considerable impact on workforce productivity and workplaces may need to have the correct preparations in place to deal with employee lateness and absenteeism due to diabetes-related complications.

As the prevalence of diabetes increases and its impact on society and the economy increases, there is a real need for clear guidelines for HCPs in Southeast Asia. Use of Western guidelines in the Southeast Asia may add to the increased diabetes burden in the region, as a result of differing disease aetiology between Western and Asian diabetes populations, such as a lower BMI and younger age at time of diabetes presentation in Asian populations. ${ }^{11}$ One of the main contributors could be the differences in diet, which is of particular importance, as a result of a higher glycemic response to carbohydrate consumption in Asian populations. $^{25}$ Other contributing factors in Asian populations include: differences in presentation of antiinsulin auto-antibodies in patients with T1D from Western compared with Asian populations, ${ }^{1,26}$ a high proportion of patients who practice Ramadan (increased risk of hyper or hypoglycaemia) and a preference for pre-mix insulin. ${ }^{11}$ Although some guidelines are available, there appears to be a difference between the guidelines and what HCPs do in practice in Southeast Asia. ${ }^{2}$ This warrants the development of clear guidelines specific to the Asian population.

In a previous study, predictors for glycaemic control in Asia were lack of microvascular complications, old age, BMI $<30 \mathrm{~kg} / \mathrm{m}^{2}$ and self-adjustment of insulin doses. ${ }^{9}$ Lifestyle modifications can prevent diabetes and may have beneficial effects on cardiovascular risk factors, reduce the risk of microvascular complications, improve BMI and are cost-effective for both the patient and healthcare systems. ${ }^{9}$

Limitations to the study include the small sample size of patients with T1D, although this could reflect the smaller population of patients with T1D in general in Southeast Asia. Study bias may also have taken place, whereby patients were more likely to report hypoglycaemia after learning what the correct definition was during the study. This study may also be limited by recall bias in the retrospective period.

\section{CONCLUSION}

Through the use of the IO HAT questionnaires and patient diaries, a higher prospective than retrospective incidence of any and severe hypoglycaemia was reported in the Southeast Asian cohort of the IO HAT study. These results are consistent with those from the overall IO HAT study. As a result of lack of knowledge and awareness, any and severe hypoglycaemia may be retrospectively underestimated and under-reported by patients in this region. Patients on mixed (both short- and long-acting) insulin regimens had the highest rates of any hypoglycaemia prospectively in both T1D and T2D and hypoglycaemia rates generally increased with diabetes duration. Hypoglycaemia increased healthcare utilisation and reduced productivity. There is a need for unique guidelines, specific to the Southeast Asian population, which can help improve diabetes management and improve patient outcomes in those already diagnosed.

\section{Acknowledgments}

The authors acknowledge medical writing support provided by Cassandra Hines and Germanicus Hansa-Wilkinson of Watermeadow Medical, funded by Novo Nordisk..

\section{Statement of Authorship}

All authors certified fulfillment of ICMJE authorship criteria.

\section{Author Disclosure}

Dr. Faruque Pathan is a member of the advisory panels for Novo Nordisk; board member of Novo Nordisk, Sanofi and Eli Lilly; employee of BIRDEM General Hospital, Dhaka Bangladesh; received research support from Novo Nordisk, Sanofi and Novartis and has taken part in speakers' bureaus for Novo Nordisk, Sanofi, Novartis, Eli Lilly and Life-Scan. Dr. Su-Yen Goh is a member of the local advisory board of the following companies, and has received speaker honoraria from the following companies: Novo Nordisk, Sanofi Aventis, AstraZeneca, Boehringer Ingelheim, MSD and Eli Lilly. Drs. Anand Jain and Arvind Gadekar are employees of Novo Nordisk. Dr. Nemencio Nicodemus Jr. is a member of the local advisory board of the following companies: Novo Nordisk, AstraZeneca, Merck, Torrent; and received speaker honoraria from Novo Nordisk, AstraZeneca, Merck, Torrent, Eli Lilly, Sanofi, LRITherapharma and Servier. Dr. Achmad Rudijanto is a member of the local advisory board of Novo Nordisk, Sanofi Aventis, Eli Lilly, AstraZeneca, Novartis, and has received speaker fee from: Novo Nordisk, Eli Lilly, AstraZeneca, Sanofi Aventis, Novartis, Kalbe Farma and Servier. 


\section{Funding Source}

Financial support for the conduct of the research was provided by Novo Nordisk. Novo Nordisk was involved in the study design; collection, analysis and interpretation of data; and decision to submit the article for publication.

\section{References}

1. Ramachandran A, Snehalatha C, Ma RC. Diabetes in South-East Asia: An update. Diabetes Res Clin Pract. 2014;103(2):231-7. PMID: 24300015. https://doi.org/10.1016/j.diabres.2013.11.011.

2. Chan JC, Gagliardino JJ, Baik SH, et al. Multifaceted determinants for achieving glycemic control: The International Diabetes Management Practice Study (IDMPS). Diabetes Care. 2009;32(2):227-33. PMID: 19033410. PMCID: PMC2628684. https://doi.org/10.2337/dc08-0435.

3. International Diabetes Federation. IDF Diabetes Atlas. 7th ed. Brussels, Belgium, 2015.

4. International Diabetes Federation. IDF Diabetes Atlas. 3rd ed. Brussels, Belgium, 2006.

5. Phan TP, Alkema L, Tai ES, et al. Forecasting the burden of type 2 diabetes in Singapore using a demographic epidemiological model of Singapore. BMJ Open Diabetes Res Care. 2014;2(1):e000012. PMID: 25452860. PMCID: PMC4212579. https://doi.org/10.1136/bmjdrc-2013000012.

6. Jayawardena R, Ranasinghe P, Byrne NM, Soares MJ, Katulanda P, Hills AP. Prevalence and trends of the diabetes epidemic in South Asia: A systematic review and meta-analysis. BMC Public Health. 2012;12:380. PMID: 22630043. PMCID: PMC3447674. https://doi.org/ 10.1186/1471-2458-12-380.

7. Ramachandran A, Ma RC, Snehalatha C. Diabetes in Asia. Lancet. 2010;375(9712):408-18. PMID: 19875164. https://doi.org/10.1016/S01406736(09)60937-5.

8. Mohan V, Deepa M, Deepa R, et al. Secular trends in the prevalence of diabetes and impaired glucose tolerance in urban South India-the Chennai Urban Rural Epidemiology Study (CURES-17). Diabetologia. 2006;49(6):1175-8. PMID: 16570158. https://doi.org/10.1007/s00125006-0219-2.

9. Ramachandran A, Snehalatha C, Samith Shetty A, Nanditha A. Primary prevention of Type 2 diabetes in South Asians - challenges and the way forward. Diabet Med. 2013;30(1):26-34. PMID: 22827704. https://doi.org/10.1111/j.1464-5491.2012.03753.x.

10. Hu EA, Pan A, Malik V, Sun Q. White rice consumption and risk of type 2 diabetes: Meta-analysis and systematic review. BMJ. 2012;344:e1454. PMID: 22422870. PMCID: PMC3307808.

11. Goh SY, Hussein Z, Rudijanto A. Review of insulin-associated hypoglycemia and its impact on the management of diabetes in South East Asian countries. J Diabetes Investig. 2017;8(5):635-45. PMID: 28236664. PMCID: PMC5584309. https://doi.org/10.1111/jdi.12647.

12. Elliott L, Fidler C, Ditchfield A, Stissing T. Hypoglycemia event rates: A comparison between real-world data and randomized controlled trial populations in insulin-treated diabetes. Diabetes Ther. 2016;7(1):45-60. PMID: 26886441. PMCID: PMC4801820. https://doi. org/10.1007/s13300-016-0157-z.

13. Edridge $\mathrm{CL}$, Dunkley AJ, Bodicoat DH, et al. Prevalence and incidence of hypoglycaemia in 532,542 people with type 2 diabetes on oral therapies and insulin: A systematic review and meta-analysis of population based studies. PLoS One. 2015;10(6):e0126427. PMID: 26061690. PMCID: PMC4465495. https://doi.org/10.1371/journal. pone. 0126427
14. Khunti K, Alsifri S, Aronson R, et al. Rates and predictors of hypoglycaemia in 27585 people from 24 countries with insulintreated type 1 and type 2 diabetes: The global HAT study. Diabetes Obes Metab. 2016;18(9):907-15. PMID: 27161418. PMCID: PMC5031206. https://doi.org/10.1111/dom.12689.

15. Emral R, Pathan F, Yepes Cortés CAY, et al. Self-reported hypoglycemia in insulin-treated patients with diabetes: Results from an international survey on 7289 patients from nine countries. Diabetes Res Clin Pract. 2017;134:17-28. PMID: 28951336. https://doi.org/10.1016/j.diabres.2017.07.031.

16. Workgroup on Hypoglycemia, American Diabetes Association. Defining and reporting hypoglycemia in diabetes: A report from the American Diabetes Association Workgroup on Hypoglycemia. Diabetes Care. 2005;28(5):1245-9. PMID: 15855602.

17. Pedersen-Bjergaard U, Pramming S, Heller SR, et al. Severe hypoglycaemia in 1076 adult patients with type 1 diabetes: Influence of risk markers and selection. Diabetes Metab Res Rev. 2004;20(6):47986. PMID: 15386817. https://doi.org/10.1002/dmrr.482.

18. Kalra S, Mukherjee JJ, Venkataraman S, et al. Hypoglycemia: The neglected complication. Indian J Endocrinol Metab. 2013;17(5):819-34.

19. Peyrot M, Barnett AH, Meneghini LF, Schumm-Draeger PM. Factors associated with injection omission/non-adherence in the Global Attitudes of Patients and Physicians in Insulin Therapy study. Diabetes Obes Metab. 2012;14(12):1081-7. PMID: 22726104. https://doi.org/10.1111/j.1463-1326.2012.01636.x.

20. Shariful Islam SM, Lechner A, Ferrari U, et al. Healthcare use and expenditure for diabetes in Bangladesh. BMJ Global Health. 2017;2(1):e000033. PMID: 28588991 PMCID: PMC5321382. https://doi.org/10.1136/bmjgh-2016-000033.

21. Khunti K, Alsifri S, Aronson R, et al. Impact of hypoglycaemia on patient-reported outcomes from a global, 24-country study of 27,585 people with type 1 and insulin-treated type 2 diabetes. Diabetes Res Clin Pract. 2017;130:121-9. PMID: 28602812. https://doi.org/10.1016/j.diabres.2017.05.004.

22. McQueen RB, Ellis SL, Campbell JD, Nair KV, Sullivan PW. Costeffectiveness of continuous glucose monitoring and intensive insulin therapy for type 1 diabetes. Cost Eff Resour Alloc. 2011;9:13. PMID: 21917132 PMCID: PMC3180394 https://doi.org/10.1186/1478-7547-9-13.

23. Tunis SL, Minshall ME. Self-monitoring of blood glucose (SMBG) for type 2 diabetes patients treated with oral anti-diabetes drugs and with a recent history of monitoring: Cost-effectiveness in the US. Curr Med Res Opin. 2010;26(1):151-62. PMID: 19919376. https://doi.org/ 10.1185/03007990903400071.

24. Pollock RF, Valentine WJ, Goodall G, Brändle M. Evaluating the costeffectiveness of self-monitoring of blood glucose in type 2 diabetes patients on oral anti-diabetic agents. Swiss Med Wkly. 2010;140:w13103. PMID: 21110238. https://doi.org/10.4414/smw.2010.13103.

25. Chen W, Qian L, Watada H, et al. Impact of diet on the efficacy of insulin lispro mix 25 and insulin lispro mix 50 as starter insulin in East Asian patients with type 2 diabetes: Subgroup analysis of the comparison between low mixed insulin and mid mixed insulin as starter insulin for patients with type 2 diabetes mellitus (CLASSIFY Study) randomized trial. J Diabetes Investig. 2017;8(1):75-83. PMID: 27287069. PMCID: PMC5217926. https://doi.org/10.1111/jdi.12547.

26. Liao $Y$, Xiang $Y$, Zhou Z. Diagnostic criteria of latent autoimmune diabetes in adults (LADA): A review and reflection. Front Med. 2012;6(3):243-7. PMID: 22843304. https://doi.org/10.1007/s11684-0120201-y.

Authors are required to accomplish, sign and submit scanned copies of the JAFES Author Form consisting of: (1) the Authorship Certification that the manuscript has been read and approved by all authors, and that the requirements for authorship have been met by each author, (2) the Author Declaration that the article represents original material that is not being considered for publication or has not been published or accepted for publication elsewhere, (3) the Statement of Copyright Transfer[accepted manuscripts become the permanent property of the JAFES and are licensed with an Attribution-Share Alike-Non-Commercial Creative Commons License. Articles may be shared and adapted for non-commercial purposes as long as they are properly cited], (4) the Statement of Disclosure that there are no financial or other relationships that might lead to a conflict of interest. For Original Articles involving human participants, authors are required to submit a scanned copy of the Ethics Review Approval of their research. For manuscripts reporting data from studies involving animals, authors are required to submit a scanned copy of the Institutional Animal Care and Use Committee approval. For Case Reports or Series, and Images in Endocrinology, consent forms, are required for the publication of information about patients; otherwise, authors declared that all means have been exhausted for securing such consent. Articles and any other material published in the JAFES represent the work of the author(s) and should not be construed to reflect the opinions of the Editors or the Publisher. 